\title{
THE EQUIVALENT LOADING METHOD AND THE EQUIVALENT BEAM METHOD ${ }^{1}$
}

\author{
BY \\ PEI-PING CHEN \\ Brown University
}

Introduction. Even under the usual engineering assumptions, the analysis of beams with non-uniform cross section subjected to distributed lateral loads, axial forces, and elastic support action is often difficult. It is the purpose of this paper to present a technique by which one can reduce more complicated beam problems to standard ones. This technique consists of two successive methods which we call the equivalent loading method and the equivalent beam method.

In the equivalent loading method an arbitrary distribution of lateral loading on a portion of a beam is replaced by concentrated loads and couples so chosen that they are entirely equivalent to the given loading so far as the remainder of the beam is concerned. Thus a simple equivalent loading of concentrated forces and couples may be used to evaluate rigorously the stress and deflection that the given loading over a portion of a beam produces in the remainder of the beam.

In an analogous fashion, the equivalent beam method is used to replace a portion of a beam by an equivalent beam portion in such a way that the stresses and deflections in the remainder of the beam are not affected by the change. In this method the original portion may be of non-uniform cross section and may have axial forces and elastic support, but must be free from lateral load; the equivalent beam portion can always be chosen to consist of segments having uniform cross sections, free from axial forces and elastic restraint.

It is assumed everywhere in the paper that the axial forces are such that elastic instability does not occur.

\section{PART I: THE EQUIVALENT LOADING METHOD Structurally balanced loading systems}

1. Fundamental fixed-ended beam problem. The equivalent loading method, which will be introduced in this paper to analyze complicated beam problems, depends on the analysis of the fundamental problem of the bending of a fixed-ended beam. The reason for this will be shown in Sec. 7 .

Let us consider a fixed-ended beam of non-uniform cross section subjected to arbitrary distribution of both lateral load and axial force (see Fig. 1); furthermore, for generality, we will assume the beam to be resting on an elastic foundation. The deflection function $y(x)$ is governed by the differential equation,

$$
L[y]=-\left\{E I(x) y^{\prime \prime}\right\}^{\prime \prime}-\left\{P(x) y^{\prime}\right\}^{\prime}-k(x) y=F(x)
$$

${ }^{1}$ Received May 28, 1948. This paper was submitted in partial fulfilment of the requirements for the degree of Master of Science in the Graduate Division of Applied Mathematics at Brown University, June, 1948. 
together with the boundary conditions

$$
y(0)=y(l)=y^{\prime}(0)=y^{\prime}(l)=0
$$

where $E I(x)$ is the rigidity, always positive and finite,

$P(x)$ is the axial compression,

$k(x)$ is the foundation reaction intensity due to unit deflection $(k(x) \geqq 0$, if the beam is elastically supported),

and $F(x)$ is the lateral load distribution (continuous or discontinuous).

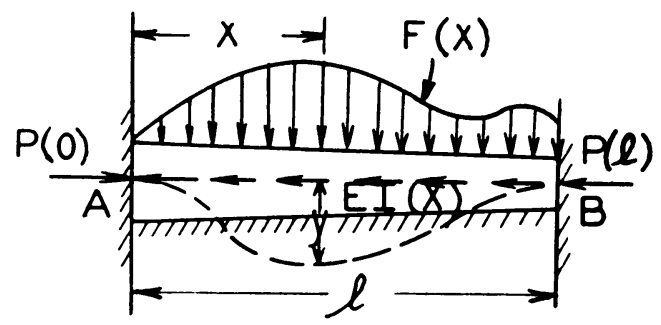

FIG. 1.

Here, as in all of the following discussion, the magnitude of the axial force is assumed to be such that buckling will not occur. The known functions, $P(x)$ and $E I(x)$, are also assumed to be piecewise differentiable and twice differentiable, respectively, in the open interval $(0, l)$.

In the derivation of Eq. (1.1), the following well-known formulas

$$
M(x)=-E I(x) y^{\prime \prime}(x), \quad S(x)=M^{\prime}(x)-P(x) y^{\prime}(x)
$$

are used for the bending moments and shear forces. The sign convention used is shown in Fig. 2.

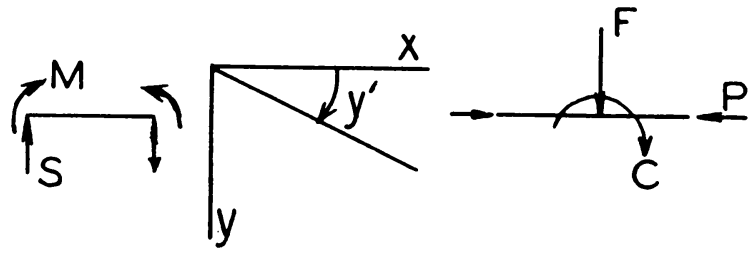

FIG. 2.

The solution of Eq. (1.1) together with the boundary conditions (1.2) may be expressed in terms of Green's function $G(x, \xi)$ :

$$
y(x)=\int_{0}^{l} G(x, \xi) F(\xi) d \xi .
$$

Here $G(x, \xi)$ is the Green's function for the operator $L[y]$ with the boundary condition (1.2); stated in physical terms, it is the deflection at $x$ due to unit lateral load at $\xi$, with the effects of both axial force as well as the elastic foundation taken into account (see Fig. 3).

\section{Structurally balanced loading system.}

Definition. A loading system $f(x)$ is said to be structurally balanced with respect to a fixed-ended beam $A B$, if and only if it produces no reaction forces and couples at 
both ends. It follows from Eqs. (1.2) and (1.3) that these reaction forces and couples vanish if and only if

$$
y^{\prime \prime}(0)=y^{\prime \prime}(l)=y^{\prime \prime \prime}(0)=y^{\prime \prime \prime}(l) .=0,
$$

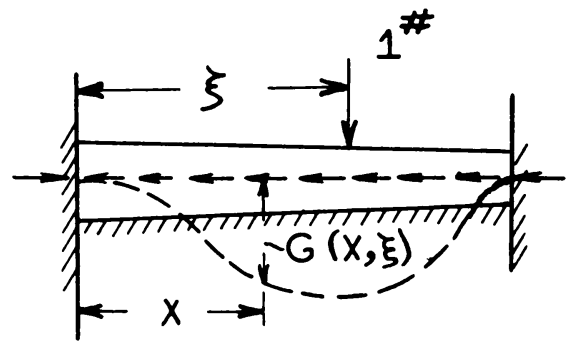

Fig. 3.

whence, from Eq. (1.4),

$$
\begin{aligned}
& \int_{0}^{l} G_{x x}(0, \xi) f(\xi) d \xi=0 \\
& \int_{0}^{l} G_{x x}(l, \xi) f(\xi) d \xi=0 \\
& \int_{0}^{l} G_{x x x}(0, \xi) f(\xi) d \xi=0 \\
& \int_{0}^{l} G_{x x x}(l, \xi) f(\xi) d \xi=0 .
\end{aligned}
$$

and

It is shown in Sec. 4 that the functions

$$
G_{x x}(0, \xi), \quad G_{x x}(l, \xi), \quad G_{x x x}(0, \xi), \quad G_{x x x}(l, \xi)
$$

must be linearly independent.

The above equations (2.2) constitute necessary and sufficient conditions for structurally balanced loading systems. These conditions might be further simplified by the aid of a linear transformation (see Example I).

EXAMPLE I: To find conditions which must be satisfied by all structurally balanced loading systems acting on a fixed-ended uniform beam under the following conditions:

$$
E I(x)=\text { constant }, \quad P(x)=k(x)=0 .
$$

SOLUTION: The Green's function is found to be:

$$
G(x, \xi)= \begin{cases}x^{2}(l-\xi)^{2}(3 \xi l-x l+2 x \xi) / 6 E I l^{3}, & x<\xi \\ \xi^{2}(l-x)^{2}(3 x l-\xi l+2 \xi x) / 6 E I l^{3}, & \xi<x\end{cases}
$$


from which we have

$$
\begin{aligned}
& G_{x x}(0, \xi)=\xi(l-\xi)^{2} / E I l^{2}, \\
& G_{x x}(l, \xi)=\xi^{2}(l-\xi) / E I l^{2}, \\
& G_{x x x}(0, \xi)=(l-\xi)^{2}(2 \xi-l) / E I l^{3},
\end{aligned}
$$

and

$$
G_{x x x}(l, \xi)=\xi^{2}(3 l+2 \xi) / E I l^{3} .
$$

The above set of functions may be further simplified to the following set of linearly independent functions,

$$
1, \quad \xi, \quad \xi^{2}, \quad \xi^{3}
$$

by a linear transformation

$$
\xi=E I G_{x x}(0, \xi)+\frac{7}{5} E I G_{x x}(l, \xi)+\frac{l}{5} E I G_{x x x}(l, \xi), \quad \text { etc. }
$$

Thus, according to Eq. (2.2), necessary and sufficient conditions for a structurally balanced loading system are the following equations:

$$
\int_{0}^{l} \xi^{m} f(\xi) d \xi=0, \quad m=0,1,2,3 .
$$

\section{Auxiliary functions of a beam.}

Definition. Four functions $\varphi_{1}(\xi), \varphi_{2}(\xi), \varphi_{3}(\xi), \varphi_{4}(\xi)$ are said to form a set of auxiliary functions of a fixed-ended beam if and only if they are linearly independent and are such that the relations

$$
\int_{0}^{l} \varphi_{i}(\xi) f(\xi) d \xi=0, \quad i=1,2,3,4
$$

hold for every loading system $f(x)$ which is structurally balanced with respect to that beam $A B$. For example, the functions (2.3) form a set of auxiliary functions.

In order to find the differential equation for auxiliary functions, we rewrite Eq. (1.1) in a more general form:

$$
L[y]=\alpha(x) y^{\mathrm{iv}}+\beta(x) y^{\prime \prime \prime}+\gamma(x) y^{\prime \prime}+\delta(x) y^{\prime}+\epsilon(x) y=F(x) .
$$

For any auxiliary function $\varphi(x)$ and any arbitrary structurally balanced loading system $f(x)$, we have

$$
\begin{aligned}
0 & =\int_{0}^{l} \varphi(\xi) f(\xi) d \xi \\
& =\int_{0}^{l} \varphi(\xi)\left\{\alpha(\xi) y^{i 0}+\beta(\xi) y^{\prime \prime \prime}+\gamma(\xi) y^{\prime \prime}+\delta(\xi) y^{\prime}+\epsilon(\xi) y\right\} d \xi \\
& =\left.\left\{A y^{\prime \prime \prime}+B y^{\prime \prime}+C y^{\prime}+D y\right\}\right|_{\xi=0} ^{\xi=l}+\int_{0}^{l} y(\xi) L^{*}[\varphi(\xi)] d \xi
\end{aligned}
$$


by integration by parts where $A=\alpha(\xi) \varphi(\xi), B=-\{\alpha(\xi) \varphi(\xi)\}^{\prime}+\beta(\xi) \varphi(\xi)$; etc., and $L^{*}[\varphi]=\{\alpha(\xi) \varphi\}^{\text {iv }}-\{\beta(\xi) \varphi\}^{\prime \prime \prime}+\{\gamma(\xi) \varphi\}^{\prime \prime}-\{\delta(\xi) \varphi\}^{\prime}+\epsilon(\xi) \varphi$. This last operator $L^{*}$ is usually called the adjoint operator of $L$. Since $y=y^{\prime}=y^{\prime \prime}=y^{\prime \prime \prime}=0$ at both ends (see Eqs. (1.2) and (2.1)), we get

$$
\int_{0}^{l} y(\xi) L^{*}[\varphi(\xi)] d \xi=0
$$

for which a sufficient condition is

$$
L^{*}[\varphi(\xi)]=0 .
$$

To show that Eq. (3.4) is also necessary for an auxiliary function $\varphi(\xi)$, we assume that $L^{*}[\varphi(\xi)]>0$ at some point $\xi$. By continuity, there exists a neighborhood of $\xi$,

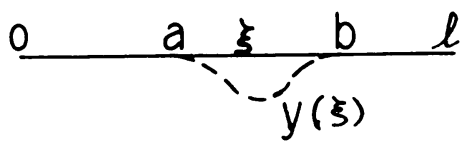

Fig. 4.

say $(a, b)$, such that $L^{*}[\varphi(\xi)]>0$ for $\xi$ in $(a, b)$. Let us construct a deflection function $y(x)$ defined by (see Fig. (4)):

$$
\left\{\begin{array}{lll}
y=0 & \text { when } & x \text { is not in }(a, b) \\
y>0 & \text { when } & x \text { is in }(a, b)
\end{array}\right.
$$

so that Eqs. (1.2) and (2.1) are satisfied, but not Eq. (3.3). Then there exists a structurally balanced loading system $F(x)$ obtainable from Eq. (3.2) and Eq. (3.5) and an auxiliary function $\varphi(\xi)$ such that

$$
\int_{0}^{l} \varphi(\xi) F(\xi) d \xi=\int_{0}^{l} y(\xi) L^{*}[\varphi(\xi)] d \xi \neq 0
$$

which contradicts Eq. (3.3). Hence the necessity of Eq. (3.4) is proved.

Since the operator $L$ defined by Eq. (1.1) is self-adjoint, any set of linearly independent complementary functions ${ }^{2}$ of that equation may be used as a set of auxiliary functions.

4. Necessary and sufficient conditions for structurally balanced loading systems in terms of auxiliary functions. Necessary and sufficient conditions for structurally balanced loading systems have already been obtained in terms of Green's function (see Eq. (2.2)). Now we try to express them in terms of auxiliary functions.

From the definition of auxiliary functions, it is seen that

$$
\int_{0}^{l} \varphi_{i}(\xi) f(\xi) d \xi=0, \quad i=1,2,3,4
$$

are necessary conditions for a structurally balanced loading system $f(x)$. Here the $\varphi_{i}(\xi)$ are the linearly independent complementary functions of Eq. (1.1).

${ }^{2}$ E. L. Ince, Ordinary differential equations, Dover Publications, New York, 1944, p. 402. 
To prove the sufficiency of Eq. (4.1), that is, to show that a loading system $f(x)$ is structurally balanced if it satisfies Eq. (4.1), we need to have the following additional structural stability condition:

$$
\Delta[\varphi]=\left|\begin{array}{llll}
\varphi_{1}(0) & \varphi_{1}(l) & \varphi_{1}^{\prime}(0) & \varphi_{1}^{\prime}(l) \\
\varphi_{2}(0) & \varphi_{2}(l) & \varphi_{2}^{\prime}(0) & \varphi_{2}^{\prime}(l) \\
\varphi_{3}(0) & \varphi_{3}(l) & \varphi_{3}^{\prime}(0) & \varphi_{3}^{\prime}(l) \\
\varphi_{4}(0) & \varphi_{4}(l) & \varphi_{4}^{\prime}(0) & \varphi_{4}^{\prime}(l)
\end{array}\right| \neq 0 .
$$

For if any loading system $f(x)$ satisfies the conditions (4.1), then by repeating the the argument of Sec. 3, we obtain

$$
\left.\left\{A_{i}(\xi) y^{\prime \prime \prime}(\xi)+B_{i}(\xi) y^{\prime \prime}(\xi)\right\}\right|_{\xi=0} ^{\xi=l}=0, \quad i=1,2,3,4
$$

due to the facts

(i) that $y(0)=y(l)=y^{\prime}(0)=y^{\prime}(l)=0$ for a fixed-ended beam, and (ii) that $L^{*}\left[\varphi_{i}(\xi)\right]=0$ obtained from the given condition (4.1).

ince the determinant of the coefficients of $y^{\prime \prime \prime}(0), y^{\prime \prime \prime}(l), y^{\prime \prime}(0)$ and $y^{\prime \prime}(l)$ in Eq. (4.3),

$$
\left|\begin{array}{llll}
A_{1}(0) & A_{1}(l) & B_{1}(0) & B_{1}(l) \\
A_{2}(0) & A_{2}(l) & B_{2}(0) & B_{2}(l) \\
A_{3}(0) & A_{3}(l) & B_{3}(0) & B_{3}(l) \\
A_{4}(0) & A_{4}(l) & B_{4}(0) & B_{4}(l)
\end{array}\right|=\{E I(0) E I(l)\}^{2} \Delta[\varphi],
$$

does not vanish by the stability condition (4.2), we get Eq. (2.1). Thus $f(x)$ is a structurally balanced loading system.

It is interesting to point out from the above argument that the functions (2.3) are also linearly independent and they exist only when the stability condition (4.2) holds. EXAMPLE II: To find the auxiliary functions for a fixed-ended uniform beam column under the following condition:

$$
E I(x)=\text { constant }, \quad P(x)=\text { constant }>0, \quad k(x)=0 .
$$

SOLUTION: The differential equation (1.1) becomes

$$
-E I y^{\mathrm{iv}}-P y^{\prime \prime}=F(x) .
$$

Hence the auxiliary functions are

$$
1, \quad \xi, \quad \sin \xi / j, \quad \cos \xi / j,
$$

respectively, where $j^{2}=E I / P$. And the stability condition (4.2) becomes

$$
\Delta[\varphi]=\frac{4}{j} \sin \frac{l}{2 j}\left(\sin \frac{l}{2 j}-\frac{l}{2 j} \cos \frac{l}{2 j}\right) \neq 0
$$

which must hold for a stable fixed-ended beam column. 


\section{Structurally equivalent loading systems}

\section{Structurally equivalent loading systems.}

Definition. Two loading systems, $f(x)$ and $g(x)$, are said to be structurally equivalent with respect to a fixed-ended beam $A B$ if and only if they give the same reaction system at both ends (in other words, the difference, $f(x)-g(x)$, forms a structurally balanced loading system with respect to the given beam).

Generalizing this definition, two loading systems are said to be structurally equivalent with respect to a portion $A B$ of a beam if they are structurally equivalent with respect to the fixed-ended beam obtained by clamping the points $A$ and $B$. To be sure that two loading systems, $f(x)$ and $g(x)$, are structurally equivalent, we need ${ }^{3}$

$$
\int_{0}^{l} \varphi_{i}(\xi) f(\xi) d \xi=\int_{0}^{l} \varphi_{i}(\xi) g(\xi) d \xi, \quad i=1,2,3,4
$$

where the $\varphi_{i}$ are auxiliary functions. It is understood that the stability condition (4.2) is satisfied.

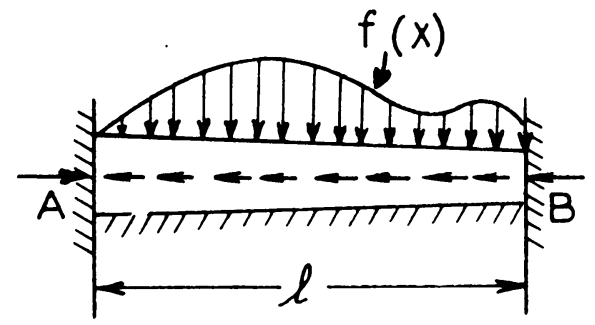

Fig. 5a.

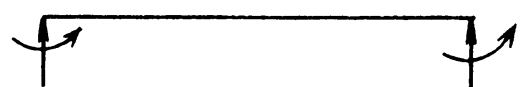

FIG. $5 b$.

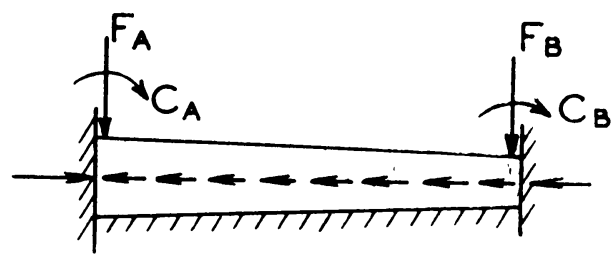

FIG. 5c.

6. Reversed reaction system. Fig. 5a shows a given loading system $f(x)$, acting on a fixed-ended beam $A B$. The loading system produces a reaction system as shown in Fig. 5b. Then, by definition, the reversed reaction system (Fig. 5c) also constitutes a loading system structurally equivalent to $f(x)$. Thus it is seen that there exists at least one loading system which is structurally equivalent to a given loading system-namely, the reversed reaction system. However, in some special cases, other useful types of structurally equivalent loading systems might be more easily obtained (see Fig. 6b).

It will now be shown how the auxiliary functions may be used to determine the

${ }^{3}$ The following facts are noted:

(1) If the loading $g(x)$ consists of a concentrated load $F$ at $\xi$, the right-hand side of Eq. (5.1) becomes $\varphi_{i}(\xi) F$.

(2) If the loading $g(x)$ consists of a concentrated couple $C$ at $\xi$, the right-hand side of Eq. (5.1) becomes $\varphi^{\prime}{ }_{i}(\xi) C$; this can easily be shown by using two concentrated loads $-C / \epsilon$ and $+C / \epsilon$ at $\xi$ and $\xi+\epsilon$, respectively, and then letting $\epsilon \rightarrow 0$. 
reversed reaction system. If $\varphi_{i}(\xi)$ is a set of auxiliary functions and if $\Delta[\varphi] \neq 0$, then Eq. (5.1) gives ${ }^{3}$

$$
\begin{aligned}
& F_{A}^{\prime} \varphi_{i}(0)+F_{B} \varphi_{i}(l)+C_{A} \varphi_{i}^{\prime}(0)+C_{B} \varphi_{i}^{\prime}(l) \\
& =\int_{0}^{l} \varphi_{i}(\xi) f(\xi) d \xi=\eta_{i}, \quad \text { say, }, \quad i=1,2,3,4 .
\end{aligned}
$$

Now if the $\varphi_{i}$ are chosen so that

$$
\left.\begin{array}{l}
\varphi_{2}(0)=\varphi_{3}(0)=\varphi_{4}(0)=\varphi_{3}(l)=\varphi_{4}(l)=\varphi_{4}^{\prime}(0)=0, \\
\varphi_{1}(0)=\varphi_{2}(l)=\varphi_{3}^{\prime}(0)=\varphi_{4}^{\prime}(l)=\Delta[\varphi]=1,
\end{array}\right\}
$$

then the reversed reaction system may be readily found to be

$$
\begin{aligned}
& C_{B}=\eta_{4}, C_{A}=\left|\begin{array}{cc}
\eta_{3} & \varphi^{\prime}(l) \\
\eta_{4} & 1
\end{array}\right|, \\
& \vec{F}_{B}=\left|\begin{array}{ccc}
\eta_{2} & \varphi_{2}^{\prime}(0) & \varphi_{2}^{\prime}(l) \\
\eta_{3} & 1 & \varphi_{3}^{\prime}(l) \\
\eta_{4} & 0 & 1
\end{array}\right|, \quad F_{A}=\left|\begin{array}{cccc}
\eta_{1} & \varphi_{1}(l) & \varphi_{1}^{\prime}(0) & \varphi_{1}^{\prime}(l) \\
\eta_{2} & 1 & \varphi_{2}^{\prime}(0) & \varphi_{2}^{\prime}(l) \\
\eta_{3} & 0 & 1 & \varphi_{3}^{\prime}(l) \\
\eta_{4} & 0 & 0 & 1
\end{array}\right| .
\end{aligned}
$$

The above choice of $\varphi_{i}$ is made possible by using the following linear transformation process on any given set of auxiliary functions $\psi_{1}, \psi_{2}, \psi_{3}$ and $\psi_{4}$ with $\Delta[\psi] \neq 0$ :

$$
\begin{aligned}
& \varphi_{1}(\xi)=\psi_{1}(\xi) / \psi_{1}(0), \quad \varphi_{2}(\xi)=\left|\begin{array}{lll}
\psi_{1}(0) & \psi_{1}(\xi) \\
\psi_{2}(0) & \psi_{2}(\xi)
\end{array}\right| \div\left|\begin{array}{ll}
\psi_{1}(0) & \psi_{1}(l) \\
\psi_{2}(0) & \psi_{2}(l)
\end{array}\right| \\
& \varphi_{3}(\xi)=\left|\begin{array}{lll}
\psi_{1}(0) & \psi_{1}(l) & \psi_{1}(\xi) \\
\psi_{2}(0) & \psi_{2}(l) & \psi_{2}(\xi) \\
\psi_{3}(0) & \psi_{3}(l) & \psi_{3}(\xi)
\end{array}\right| \div\left|\begin{array}{lll}
\psi_{1}(0) & \psi_{1}(l) & \psi_{1}^{\prime}(0) \\
\psi_{2}(0) & \psi_{2}(l) & \psi_{2}^{\prime}(0) \\
\psi_{3}(0) & \psi_{3}(l) & \psi_{3}^{\prime}(0)
\end{array}\right| \\
& \varphi_{4}(\xi)=\frac{1}{\Delta[\psi]} \cdot\left|\begin{array}{llll}
\psi_{1}(0) & \psi_{1}(l) & \psi_{1}^{\prime}(0) & \psi_{1}^{\prime}(\xi) \\
\psi_{2}(0) & \psi_{2}(l) & \psi_{2}^{\prime}(0) & \psi_{2}^{\prime}(\xi) \\
\psi_{3}(0) & \psi_{3}(l) & \psi_{3}^{\prime}(0) & \psi_{3}^{\prime}(\xi) \\
\psi_{4}(0) & \psi_{4}(l) & \psi_{4}^{\prime}(0) & \psi_{4}^{\prime}(\xi)
\end{array}\right|
\end{aligned}
$$

Since $\Delta[\psi] \neq 0$, all denominators in Eq. (6.4) are different from zero if a proper choice of indices 1, 2, 3 and 4 is used. Thus the existence of a reversed reaction system is assured (as is physically obvious for a stable beam). 
EXAMPLE III: Given a tapered beam problem with the following conditions:

$$
E I(x) \neq \text { constant, } \quad P(x)=k(x)=0,
$$

it is required (i) to find the auxiliary functions,

(ii) to find the reversed reaction system due to a unit load at $\xi$, and

(iii) to investigate some sufficient conditions for

a) structurally balanced loading systems, and

b) structurally equivalent loading systems

in a special case in which the reciprocal of $E I(\xi)$ is a polynomial in $\xi$.

SOLUTION: (i) The Eq. (1.1) becomes

$$
-\left[E I(x) y^{\prime \prime}\right]^{\prime \prime}=F(x) .
$$

Hence the auxiliary functions are

$$
\psi_{1}=1, \quad \psi_{2}=\xi, \quad \psi_{3}=S(\xi), \quad \psi_{4}=T(\xi)
$$

where

$$
\begin{aligned}
& S(\xi)=\frac{1}{l} \int_{0}^{\xi} \int_{0}^{\xi} H(\xi) d \xi d \xi, \\
& T(\xi)=\frac{1}{l^{2}} \int_{0}^{\xi} \int_{0}^{\xi} \xi H(\xi) d \xi d \xi,
\end{aligned}
$$

and

$$
H(\xi)=1 / E I(\xi)
$$

with the value of $\Delta[\psi]=-\lambda / l \neq 0$ where $\lambda$ is defined later in Eq. (6.9).

(ii) In order to find the reversed force system, we use the following auxiliary functions (according to Eq. (6.4)):

$$
\begin{aligned}
\varphi_{1} & =1, \quad \varphi_{2}=\xi / l, \quad \varphi_{3}=\xi-\frac{l S(\xi)}{p-q}, \\
\varphi_{4} & =-\frac{l}{\lambda}[(p-q) T(\xi)-(q-r) S(\xi)]
\end{aligned}
$$

where the quantities $p, q, r$ and $\lambda$ detined by

$$
\begin{aligned}
& p=\int_{0}^{l} H(\xi) d \xi, \quad q=\frac{1}{l} \int_{0}^{l} \xi H(\xi) d \xi \\
& r=\frac{1}{l^{2}} \int_{0}^{l} \xi^{2} H(\xi) d \xi, \quad \lambda=p r-q^{2}
\end{aligned}
$$

are all positive and finite. In this example the given loading system is a unit load at $\xi$, i.e.,

$$
f(x)=\left\{\begin{array}{lll}
0 & \text { when } & x \neq \xi, \\
1 & \text { when } & x=\xi .
\end{array}\right.
$$

Hence we have $\eta_{i}=\varphi_{i}(\xi)$. Thus from Eq. (6.3), we obtain the reversed reaction system due to a unit load at $\xi$ : 


$$
F_{A}=1-F_{B}, \quad F_{B}=[q S(\xi)-p T(\xi)] / \lambda
$$

and

$$
C_{A}=\xi-F_{B} l-C_{B}, \quad C_{B}=\varphi_{4} .
$$

(iiia) If $H(\xi)$, the reciprocal of $E I(\xi)$, can be expressed by a polynomial of degree $n$ in $\xi$, viz.,

$$
H(\xi)=\sum_{m=0}^{n} h_{m}(\xi / l)^{m},
$$

then $S(\xi)$ and $T(\xi)$ are exactly polynomials of degrees $(n+2)$ and $(n+3)$ respectively. Hence under the conditions (6.5) and (6.12), we have the following sufficient conditions that a loading system $f(x)$ be structurally balanced:

$$
\int_{0}^{l} \xi^{m} f(\xi) d \xi=0, \quad m=0,1,2, \cdots, n+3 .
$$

For instance, if $f(\xi)$ can be expressed by the sum of Legendre's polynomials of orde ${ }^{r}$ $(n+4)$ or higher, it constitutes a balanced loading system with respect to this fixedended tapered beam.

(iiib) Under the same conditions (6.5) and (6.12) as mentioned above, we may also have the following sufficient conditions for the structural equivalence of the loading systems $f(x)$ and $g(x)$ :

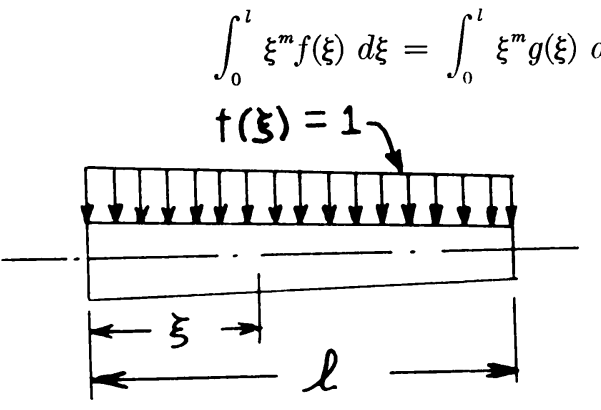

Fig. 6a.

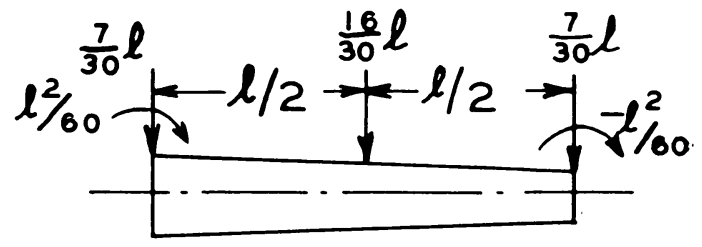

FIG. 6b.

For example, if $H(\xi)$ is a polynomial of second degree as given by

$$
H(\xi)=h_{0}+h_{1}(\xi / l)+h_{2}(\xi / l)^{2}
$$

then the two loading systems shown in Figs. $6 \mathrm{a}$ and $6 \mathrm{~b}$ are structurally equivalent because they satisfy Eq. (6.14) with $\mathrm{m}=0,1,2,3,4$, and 5 (compare with the reversed reaction system shown in Fig. $6 \mathrm{c}$ for $h_{0}=1, h_{1}=0$ and $h_{2}=2$ only). A detailed discussion of various other types of structurally equivalent loading systems ${ }^{4}$ is beyond the scope of this paper.

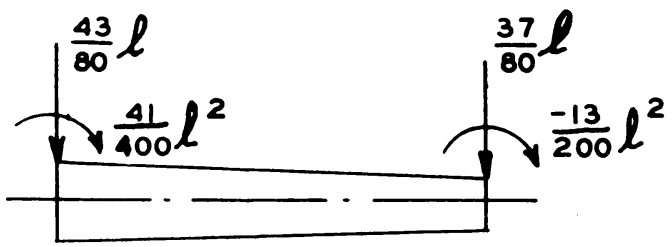

FIg. 6c.

${ }^{4}$ See, for example, P. P. Chen, Dyadic analysis of space rigid frameworks, J. Franklin Inst., Nov., 1944. 
7. The equivalent loading method. In Fig. 7a is shown a loading system $f(x)$ applied to a portion $A B$ of a beam $P Q$. Suppose that $g(x)$ is a loading system structurally equiv-
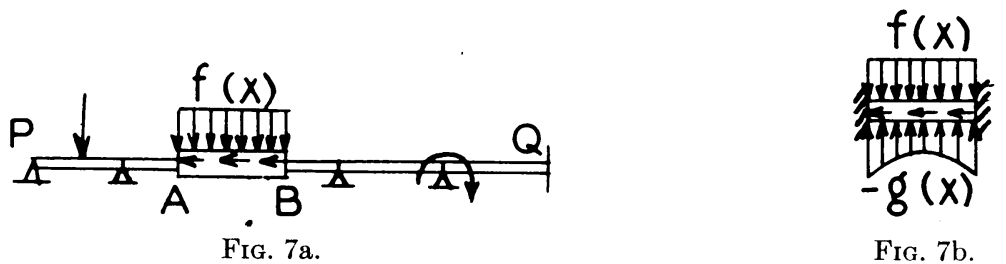

Fig. 7b.

alent to $f(x)$ with respect to this portion $A B$. By definition, if the loading systems $f(x)$ and $-g(x)$ are applied simultaneously to the fixed-ended beam $A B$ (Fig. $7 \mathrm{~b}$ ), they will produce no reaction forces and couples at the two ends $A$ and $B$. Moreover, if $f(x)$ and $-g(x)$ are applied simultaneously to the portion $A B$ of the original beam $P Q$ (Fig. 7c), they will also produce no reaction forces and couples at any support.

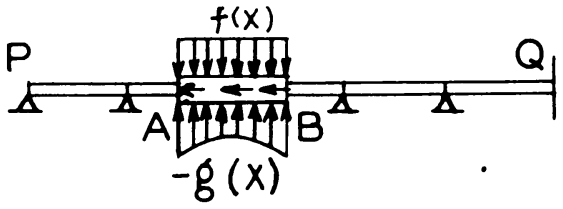

FIG. 7c.

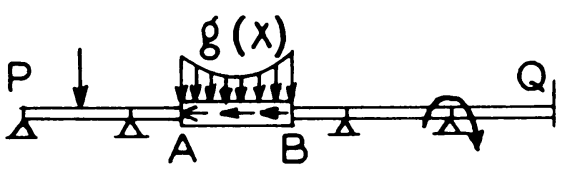

Fig. 7 d.

This follows from the fact that we may consider the portion $A B$ removed from the beam $P Q$, then loaded with the loading systems $f(x)$ and $-g(x)$, and then replaced in the beam $P Q$ without having any condition of static equilibrium or geometrical continuity violated. Now consider the beam problem shown in Fig. 7d, obtained by replacing $f(x)$ in Fig. 7a by $g(x)$. Since Fig. 7a can be obtained by superposition of Figs. $7 \mathrm{c}$ and $7 \mathrm{~d}$, and since the loadings of Fig. $7 \mathrm{c}$ produce no reaction, it follows that the loading $g(x)$ produces the same reaction forees and couples at all supports as $f(x)$ does. Thus we have

THEOREM I: A loading system $f(x)$, when applied to a portion $A B$ of a beam, may be replaced by a loading system $g(x)$, structurally equivalent with respect to the portion $A B$, without affecting the regions of the beam other than $A B$.

Thus the equivalent loading may be used to determine shear, moment, slope and deflection outside of $A B$. Within $A B$, these quantities can be found by superposition of the effects of the loadings in Fig. $7 \mathrm{c}$ and Fig. $7 \mathrm{~d}$.

\section{PART II: THE EQUIVALENT BEAM METHOD Stiffness matrix and reaction matrix}

8. Fundamental simply-supported beam problem. Just as the equivalent loading method depends on the analysis of a fixed-ended beam, the equivalent beam method depends on the analysis of a simply-supported beam. The reason for this can be seen in Sec. 12.

Let us consider a horizontal simply-supported beam $A B$ (Fig. 8) of length $l$ loaded 
by two couples $C_{A}$ and $C_{B}$. It is required to find the slopes and shears at both ends. In this case the deflection, $y(x)$, is given by

$$
L[y]=0,
$$

together with the boundary conditions

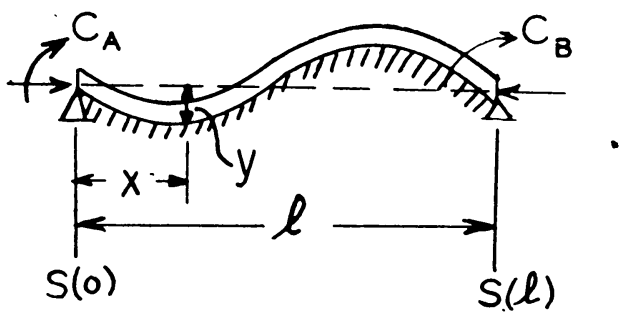

FIG. 8.

and

$$
\begin{gathered}
y(0)=y(l)=0 \\
E I(0) y^{\prime \prime}(0)=-C_{A}, \quad E I(l) y^{\prime \prime}(l)=C_{B} .
\end{gathered}
$$

The general solution may again be expressed by

$$
y(x)=\int_{0}^{l} G(x, \xi) F(\xi) d \xi
$$

where the Green's function $G(x, \xi)$, the deflection at $x$ due to a unit load at $\xi$ acting on this simply-supported beam $A B$, is assumed to exist, and the load system $F(\xi)$ is considered as the couples $C_{A}$ and $C_{B}$. Thus Eq. (8.4) gives: ${ }^{3}$

$$
y(x)=G_{\xi}(x, 0) C_{A}+G_{\xi}(x, l) C_{B} .
$$

By differentiation, we have the required end slopes:

$$
\begin{aligned}
& y^{\prime}(0)=G_{x \xi}(0,0) C_{A}+G_{x \xi}(0, l) C_{B}, \\
& y^{\prime}(l)=G_{x \xi}(l, 0) C_{A}+G_{x \xi}(l, l) C_{B},
\end{aligned}
$$

or, written in matrix form,

$$
\left[y^{\prime}(0), y^{\prime}(l)\right]=\left[C_{A}, C_{B}\right]\left[\begin{array}{ll}
G_{x \xi}(0,0) & G_{x \xi}(l, 0) \\
G_{x \xi}(0, l) & G_{x \xi}(l, l)
\end{array}\right] .
$$

In the above equation, the square matrix

$$
\left[\begin{array}{ll}
G_{x \xi}(0,0) & G_{x \xi}(l, 0) \\
G_{x \xi}(0, l) & G_{x \xi}(l, l)
\end{array}\right]=\left[\begin{array}{ll}
\alpha & \beta \\
\beta & \gamma
\end{array}\right], \quad \text { say, }
$$

which may be called the flexibility matrix, is a symmetrical one due to the fact that the Green's function is symmetrical with respect to $x$ and $\xi$. Its existence, as will be discussed later, depends on the fulfilment of the stability condition (9.6) for a simplysupported beam. However, its reciprocal matrix,

$$
\left[\begin{array}{ll}
\alpha & \beta \\
\beta & \gamma
\end{array}\right]^{-1}=\left[\begin{array}{ll}
\bar{\alpha} & \bar{\beta} \\
\bar{\beta} & \bar{\gamma}
\end{array}\right], \quad \text { say },
$$


which may be called the stiffness matrix, ${ }^{5}$ is more useful in the moment distribution method. It is also a symmetrical matrix, but its existence depends on the fulfilment of stability condition (4.2) for a fixed-ended beam instead of (9.6) which is the stability condition for a simply-supported beam.

EXAMPLE IV: For a simply-supported uniform beam under the condition (2.4), the Green's function is

$$
G(x, \xi)= \begin{cases}x(l-\xi)\left(2 l \xi-\xi^{2}-x^{2}\right) / 6 E I l, & x<\xi, \\ \xi(l-x)\left(2 l x-x^{2}-\xi^{2}\right) / 6 E I l, & \xi<x .\end{cases}
$$

Here the flexibility and stiffness matrices are, respectively,

$$
\left[\begin{array}{ll}
\alpha & \beta \\
\beta & \gamma
\end{array}\right]=\left[\begin{array}{rr}
2 & -1 \\
-1 & 2
\end{array}\right] \frac{l}{6 E I}, \quad\left[\begin{array}{ll}
\bar{\alpha} & \bar{\beta} \\
\bar{\beta} & \bar{\gamma}
\end{array}\right]=\left[\begin{array}{ll}
2 & 1 \\
1 & 2
\end{array}\right] \frac{2 E I}{l} .
$$

The end shears may be obtained from the static condition

$$
S(0)=S(l)=-\left(C_{A}+C_{B}\right) / l .
$$

9. Stiffness and reaction matrices in terms of auxiliary functions. The general solution of Eq. (8.1) may also be expressed in terms of auxiliary functions $\varphi_{i}(x)$ :

$$
y(x)=\sum_{i=1}^{4} a_{i} \varphi_{i}(x) .
$$

From Eq. (9.1) and the boundary conditions (8.2) and (8.3), the coefficients $a_{i}$ may be determined. Again if the $\varphi_{i}$ functions given by Eq. (6.4) are used, we have,

$$
\begin{aligned}
& a_{1}=a_{2}=0, \\
& y^{\prime}(0)=a_{3}, \\
& y^{\prime}(l)=a_{3} \varphi_{3}^{\prime}(l)+a_{4}, \\
& y^{\prime \prime}(0)=-C_{A} / E I(0)=a_{3} \varphi_{3}^{\prime \prime}(0)+a_{4} \varphi_{4}^{\prime \prime}(0),
\end{aligned}
$$

and

$$
y^{\prime \prime}(l)=C_{B} / E I(0)=a_{3} \varphi_{3}^{\prime \prime}(l)+a_{4} \varphi_{4}^{\prime \prime}(l),
$$

provided that the stability condition (4.2) holds. Eliminating $a_{3}$ and $a_{4}$, we have

$$
\left[C_{A}, C_{B}\right]=\left[y^{\prime}(0), y^{\prime}(l)\right]\left[\begin{array}{ll}
\bar{\alpha} & \bar{\beta} \\
\bar{\beta} & \bar{\gamma}
\end{array}\right]
$$

where the elements in the stiffness matrix are given by

$$
\begin{aligned}
& \bar{\alpha}=\left[-\varphi_{3}^{\prime \prime}(0)+\varphi_{3}^{\prime}(l) \varphi_{4}^{\prime \prime}(0)\right] E I(0), \\
& \bar{\beta}=-\varphi_{4}^{\prime \prime}(0) E I(0)=\left[\varphi_{3}^{\prime \prime}(l)-\varphi_{3}^{\prime}(l) \varphi_{4}^{\prime \prime}(l)\right] E I(l),
\end{aligned}
$$

and

$$
\bar{\gamma}=\varphi_{4}^{\prime \prime}(l) E I(l)
$$

${ }^{5}$ S. U. Benscoter, Matrix analysis of continuous beams, Trans. A. S. C. E. 112, 1109 (1947). 
The non-vanishing of the determinant of the stiffness matrix,

$$
\bar{\alpha} \bar{\gamma}-\bar{\beta}^{2}=-\left|\begin{array}{cc}
\varphi_{3}^{\prime \prime}(0) & \varphi_{3}^{\prime \prime}(l) \\
\varphi_{4}^{\prime \prime}(0) & \varphi_{4}^{\prime \prime}(l)
\end{array}\right| E I(0) E I(l) \neq 0,
$$

where the $\varphi_{i}$ are given by Eq. (6.4), or, more generally, the non-vanishing of the following determinant,

$$
D[\varphi]=\left|\begin{array}{llll}
\varphi_{1}(0) & \varphi_{1}(l) & \varphi_{1}^{\prime \prime}(0) & \varphi_{1}^{\prime \prime}(l) \\
\varphi_{2}(0) & \varphi_{2}(l) & \varphi_{2}^{\prime \prime}(0) & \varphi_{2}^{\prime \prime}(l) \\
\varphi_{3}(0) & \varphi_{3}(l) & \varphi_{3}^{\prime \prime}(0) & \varphi_{3}^{\prime \prime}(l) \\
\varphi_{4}(0) & \varphi_{4}(l) & \varphi_{4}^{\prime \prime}(0) & \varphi_{4}^{\prime \prime}(l)
\end{array}\right| \neq 0,
$$

where the $\varphi_{i}$ are linearly independent auxiliary functions, gives the stability condition for a simply-supported beam problem. ${ }^{6}$

To find the end shears, we start from Eq. (1.3),

$$
\begin{aligned}
S(x) & =-\left\{E I(x) y^{\prime \prime}(x)\right\}^{\prime}-P(x) y^{\prime}(x) \\
& =-\sum_{j=3,4} a_{i}\left\{E I(x) \varphi_{j}^{\prime \prime}(x)\right\}^{\prime}-P(x) y^{\prime}(x)
\end{aligned}
$$

where the coefficients $a_{j}$ may be obtained from Eq. (9.2):

$$
\left[a_{3}, a_{4}\right]=\left[y^{\prime}(0), y^{\prime}(l)\right]\left[\begin{array}{cc}
1 & -\varphi_{3}^{\prime}(l) \\
0 & 1
\end{array}\right] .
$$

Putting $x=0$ and $l$ respectively in Eq. (9.7), we have the end shears

where

$$
[S(0), S(l)]=\left[y^{\prime}(0), y^{\prime}(l)\right]\left[\begin{array}{ll}
\epsilon_{11} & \epsilon_{12} \\
& \\
\epsilon_{21} & \epsilon_{22}
\end{array}\right]
$$

$$
\begin{aligned}
& \epsilon_{11}=-P(0)-\left\{E I \varphi_{3}^{\prime \prime}\right\}^{\prime}(0)+\varphi_{3}^{\prime}(l)\left\{E I \varphi_{4}^{\prime \prime}\right\}^{\prime}(0), \\
& \epsilon_{12}=-\left\{E I \varphi_{3}^{\prime \prime}\right\}^{\prime}(l)+\varphi_{3}^{\prime}(l)\left\{E I \varphi_{4}^{\prime \prime}\right\}^{\prime}(l), \\
& \epsilon_{21}=-\left\{E I \varphi_{4}^{\prime \prime}\right\}^{\prime}(0), \\
& \epsilon_{22}=-P(l)-\left\{E I \varphi_{4}^{\prime \prime}\right\}^{\prime}(l) .
\end{aligned}
$$

If the relation (9.6) holds, then the end shears can also be expressed by

$$
[S(0), S(l)]=\left[C_{A}, C_{B}\right]\left[\begin{array}{ll}
\rho_{11} & \rho_{12} \\
\rho_{21} & \rho_{22}
\end{array}\right]
$$

${ }^{6}$ For example, for a stable simply-supported beam column under the condition (4.4), the stability condition (9.6) gives $D[\varphi]=-\left(l / j^{4}\right) \sin (l / j) \neq 0$. It is interesting to point out that if a portion $A B$ of a beam is such that $D[\varphi]=0$, then this portion acts as a hinged link. 
where the square matrix

$$
\left[\begin{array}{ll}
\rho_{11} & \rho_{12} \\
\rho_{21} & \rho_{22}
\end{array}\right]=\left[\begin{array}{ll}
\alpha & \beta \\
\beta & \gamma
\end{array}\right]\left[\begin{array}{ll}
\epsilon_{11} & \epsilon_{12} \\
\epsilon_{21} & \epsilon_{22}
\end{array}\right]
$$

may be called the reaction matrix.

However if the beam is under the conditions

$$
k(x)=0, \quad P(x)=\text { constant, }
$$

then we also obtain Eq. (8.10) even if the relation (9.6) does not hold. Thus the reaction matrix has the following simple form:

$$
\left[\begin{array}{ll}
\rho_{11} & \rho_{12} \\
\rho_{21} & \rho_{22}
\end{array}\right]=-\frac{1}{l}\left[\begin{array}{ll}
1 & 1 \\
1 & 1
\end{array}\right]
$$

with the distinctive properties that

(i) it depends on the length $l$ only

and (ii) all elements $\rho_{11}, \rho_{12}, \rho_{21}$ and $\rho_{22}$ are equal.

10. Simply supported beams with two or more segments under the conditions $k(x)=0$ and $P(x)=$ constant. In Fig. 9 is shown a simply-supported beam of two

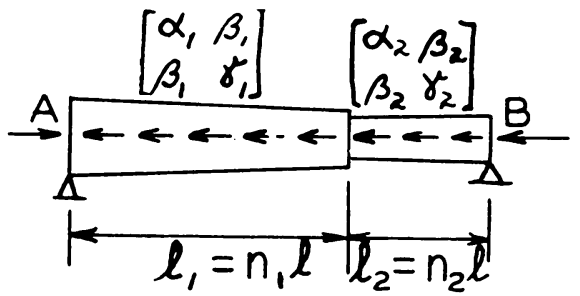

Fig. 9.

segments $A G$ and $G B$, of lengths $l_{1}$ and $l_{2}$ respectively. If the flexibility matrices are

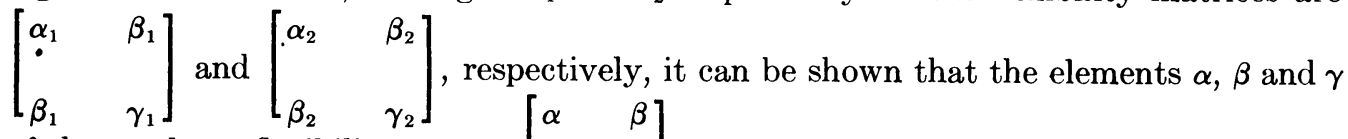
of the resultant flexibility matrix $\left[\begin{array}{ll}\alpha & \beta \\ \beta & \gamma\end{array}\right]$ of the whole beam are:

$$
\begin{aligned}
& \alpha=\alpha_{1}-2 n_{2} \beta_{1}+n_{2}^{2}\left(\gamma_{1}+\alpha_{2}\right), \\
& \beta=n_{1} \beta_{1}+n_{2} \beta_{2}-n_{1} n_{2}\left(\gamma_{1}+\alpha_{2}\right), \\
& \gamma=\gamma_{2}-2 n_{1} \beta_{2}+n_{1}^{2}\left(\gamma_{1}+\alpha_{2}\right) .
\end{aligned}
$$

and

By repeated application of Eq. (10.1), the flexibility matrix of a beam of any finite number of segments under the conditions (9.12) can be calculated.

\section{Various types of equivalent beams}

11. Equivalent beams under the conditions (9.12).

Definition. Two horizontal simply-supported beams $A B$ and $A^{\prime} B^{\prime}$ (Figs. 10a and 10b) are said to be equivalent, if and only if they have the same stiffness and reaction matrices. 
Generalizing this definition, two beam portions are said to be equivalent if they are equivalent when considered as simply-supported beams.

If these beam portions are not elastically supported, and if $P(x)=$ constant, then they need only be of equal length in order to have the same reaction matrix (see Eq. 9.13). In this paragraph we consider only this situation, unless otherwise specified.

Let us investigate the following types of equivalent beams: (Type I) Let the beam $A B$ (Fig. 10a) have a given flexibility matrix $\left[\begin{array}{ll}\alpha & \beta \\ \beta & \gamma\end{array}\right]$, and let us find an equivalent beam $A^{\prime} B^{\prime}$ (Fig. 10b) composed of two uniform segments $A^{\prime} G^{\prime}$ and $G^{\prime} B^{\prime}$ with lengths

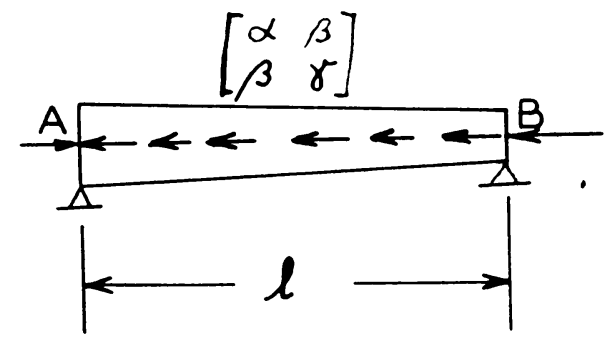

FIg. 10a.

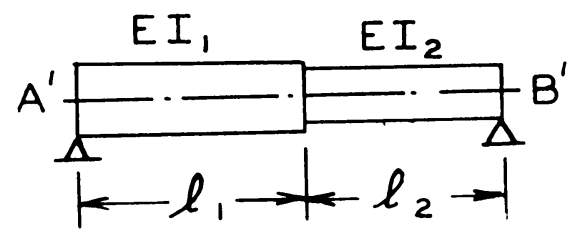

Fig. 10b.

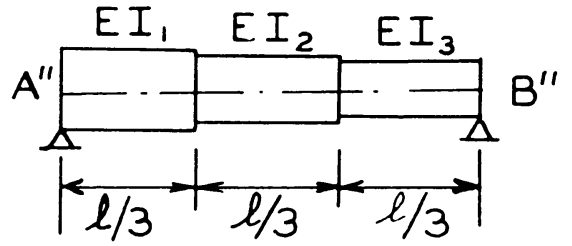

FIg. 10c.

$l_{1}$ and $l_{2}\left(=l-l_{1}\right)$ and rigidities $E I_{1}$ and $E I_{2}$ respectively. Equating the flexibility matrix of beam $A^{\prime} B^{\prime}$ (as given by Eq. (10.1)) to that of beam $A B$, we get

$$
l_{1}=\frac{\gamma+2 \beta}{\gamma-\alpha} l, \quad l_{2}=\frac{\alpha+2 \beta}{\alpha-\gamma} l
$$

and

$$
\left.E I_{1}=\frac{l}{3 \alpha+(\alpha+2 \beta) l_{2} / l_{1}}, \quad E I_{2}=\frac{l}{3 \gamma+(\gamma+2 \beta) l_{1} / l_{2}}\right)
$$

provided that $0 \leqq l_{1} \leqq l$, that is,

$$
\alpha \geqq-2 \beta \geqq \gamma .
$$

(Type II) Assume that the beam $A B$ has a given flexibility matrix $\left[\begin{array}{ll}\alpha & \beta \\ \beta & \gamma\end{array}\right]$, and find an equivalent beam $A^{\prime \prime} B^{\prime \prime}$ (Fig. 10c) composed of three parts of equal length but different rigidities $E I_{1}, E I_{2}$ and $E I_{3}$. We get

$$
\begin{aligned}
& E I_{1}=2 l /(11 \alpha+2 \gamma+14 \beta), \\
& E I_{2}=-2 l /(7 \alpha+7 \gamma+40 \beta), \\
& E I_{3}=2 l /(11 \gamma+2 \alpha+14 \beta) .
\end{aligned}
$$$$
\text { and }
$$ 
Hence under the conditions $k(x)=0$ and $P(x)=$ constant, it is always possible to find an equivalent beam composed of only three uniform segments, if the use of zero or negative values of $E I$ is permitted.

However, if (9.12) is not satisfied, the elements $\rho_{11}, \rho_{12}, \rho_{21}$ and $\rho_{22}$ in the reaction matrix are, in general, not equal to each other. Hence, we cannot find an equivalent portion $A^{\prime} B^{\prime}$ for the original portion $A B$ (Fig. 11a) unless some additional supports are provided (see Fig. 11b). A detailed discussion of equivalent beams for this case is not given here.

EXAMPLE $V$ : To find the equivalent beams for a tapered beam (Fig. 12a) under the condition (6.5).

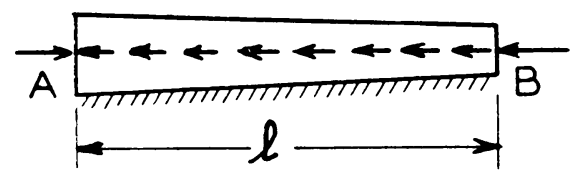

FIG. 11a.

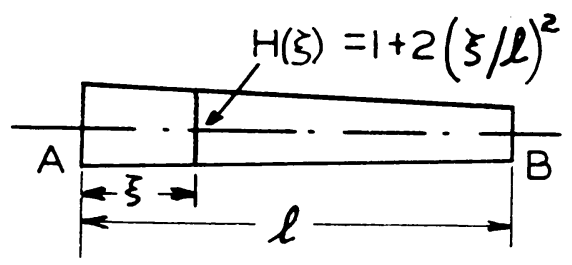

FIg. 12a.

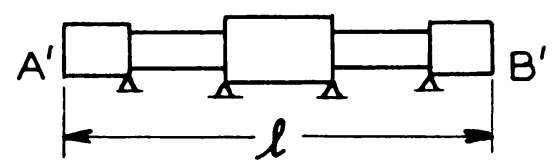

FIG. 11b.

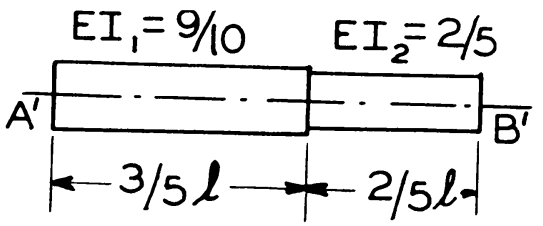

FIg. 12b.

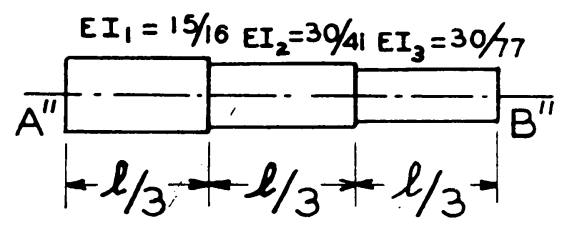

FIG. 12c.

SOLUTION: Use the auxiliary functions (6.8).

From Eqs. (9.4), (8.7) and (9.12), we have

$$
\begin{aligned}
& \bar{\alpha}=r / \lambda, \quad \bar{\beta}=(q-r) / \lambda, \quad \bar{\gamma}=(p-2 q+r) / \lambda \\
& \alpha=p-2 q+r, \quad \beta=r-q, \quad \gamma=r
\end{aligned}
$$

and

$$
\rho_{11}=\rho_{12}=\rho_{21}=\rho_{22}=-1 / l \text {. }
$$

As a numerical example, we assume that $H(\xi)$ of the beam (Fig. 12a) is given by

$$
H(\xi)=1+2(\xi / l)^{2} .
$$

From Eqs. (6.9) and (11.4), we have

$$
p=5 l / 3, \quad q=l, \quad r=\gamma=11 l / 15, \quad \alpha=2 l / 5, \quad \beta=-4 l / 15 .
$$

Then by Eqs. (11.1) and (11.3), the equivalent beams may be obtained as shown in Figs. 12b and 12c. 
12. The equivalent beam method and an illustrative example. In Fig. 13a is shown a loaded beam with a portion $A B$ which is free from lateral load. In Fig. 13b is shown the same loaded beam except that the portion $A B$ is replaced by its equivalent one, $A^{\prime} B^{\prime}$. By reasoning similar to that used in Sec. 7, we have

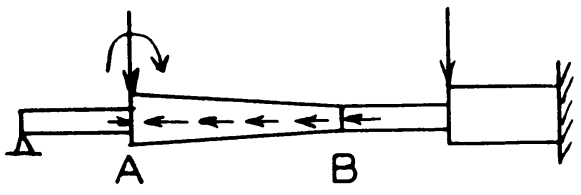

FIG. 13a.

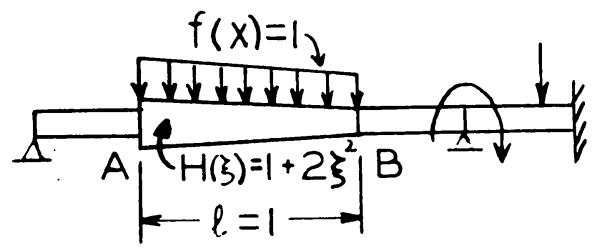

FIG. 14a.

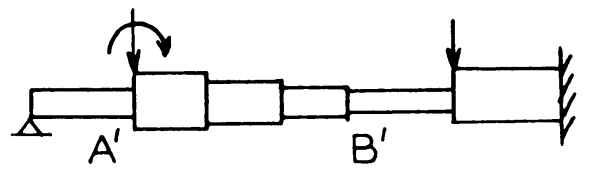

FIG. 13b.

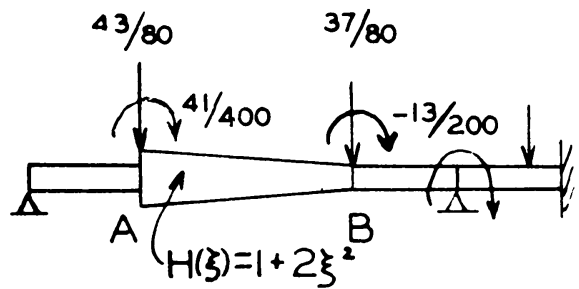

FIG. 14b.

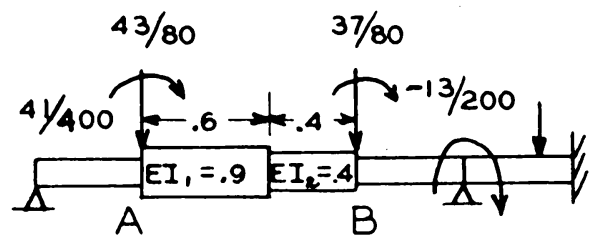

FIG. 14c.

THEOREM II. In any beam problem, a portion $A B$, if free from lateral load, may be replaced by an equivalent portion without affecting the regions of the beam other than $A B$.

As a final illustration of the method presented, we will show the application to the beam shown in Fig. 14a.

EXAMPLE VI. To simplify the beam problem shown in Fig. 14a by using both equivalent loading and equivalent beam methods (use $l=1$ for convenience).

SOLUTION: First, using the equivalent loading method to remove the distributed loads (see Figs. 6a and 6c), we get Fig. 14b.

Secondly, applying the equivalent beam method to the portion $A B$ in Fig. $14 \mathrm{~b}$ to remove the varying cross sections (see Figs. $12 \mathrm{a}$ and $12 \mathrm{~b}$ ), we get Fig. 14c. Then the analysis of the beam in Fig. 14c will give precise results for the beam in Fig. 14a everywhere other than the portion $A B$. Thus we may at least obtain the precise results at the ends $A$ and $B$ of the affected portion. These precise results might be then used in the determination of exact local value of shear, moment, slope and deflection within the portion $A B$.

By using these methods, we obtain the linearized $M / E I$ diagrams which greatly facilitate the subsequent computations. 